\title{
Commentaries
}

\section{MUNC-ing around with insulin action}

\author{
David E. James
}

\author{
Garvan Institute of Medical Research, Darlinghurst, Sydney, New South Wales, Australia.
}

\begin{abstract}
Defective uptake of glucose into muscle and fat cells, or insulin resistance, is a central feature of obesity and type 2 diabetes. As we brace ourselves for the diabetes epidemic, it is reassuring to know that real progress is being made in defining the molecular biology of how insulin stimulates glucose uptake and what goes awry in obesity and type 2 diabetes. An understanding of the molecular determinants of insulin-stimulated glucose transport has been one of the holy grails of hormone action research. A major breakthrough was the discovery that insulin stimulates the translocation of a specific glucose transport protein, GLUT4, from intracellular vesicles to the cell surface. Elucidating how this process is regulated has remained a challenge because it represents a convergence of 2 disparate and complex fields of research - namely, vesicle transport and signal transduction. A study reported in this issue of the JCI using mice lacking Munc18c, one of the vesicle-trafficking proteins involved in GLUT4 translocation, has provided new insights into the signaling/trafficking intersection that controls insulin-stimulated GLUT4 movement (see the related article beginning on page 291).
\end{abstract}

Dysregulation of glucose transport into muscle and fat cells is one of the defining features of insulin resistance. If combined with a pancreatic defect, this will lead to persistent hyperglycemia, type 2 diabetes, and its associated complications, such as eye, nerve, and kidney disease. Insulin stimulates glucose transport in muscle and fat cells by provoking translocation of the GLUT4 glucose transporter from intracellular GLUT4 storage vesicles (GSVs) to the plasma membrane (reviewed in ref. 1). Several soluble $\mathrm{N}$-ethylmaleimide-sensitive factor attachment protein receptors (SNAREs), including vesicle-associated membrane protein 2 (VAMP2), which is located on the GSV membrane, play an important role in exocytosis of GSVs. VAMP2 helps guide the vesicles to the plasma membrane docking site, which consists of the target SNAREs syntaxin 4 and $23-\mathrm{kDa}$ synaptosomal-associated protein (SNAP23) (reviewed in ref. 1). Importantly, these SNAREs are not unique to GLUT4 trafficking and likely regulate many different exocytic steps in a variety of cell types. The PI3K-Akt pathway plays an important role in insulin-stimulated

Nonstandard abbreviations used: GAP, GTPase-activating protein; GSV, GLUT4 storage vesicle; SM, Sec1p/ Munc18; SNARE, soluble $N$-ethylmaleimide-sensitive factor attachment protein receptor; VAMP2, vesicleassociated membrane protein 2 .

Conflict of interest: The author has declared that no conflict of interest exists.

Citation for this article: J. Clin. Invest. 115:219-221 (2005). doi:10.1172/JCI200524158. glucose transport in muscle and fat cells (reviewed in refs. 2, 3), and recent evidence suggests that SNAREs and their associated proteins are targets of the Akt pathway.

SNAREs are thought to play an essential role in both the high-affinity encounter between a transport vesicle and its target membrane and the subsequent fusion reaction that pulls the 2 membranes together (Figure 1 and refs. 4, 5). SNAREs can form a core complex consisting of 4 different $\alpha$ helices from each of the constituent SNARE proteins. A major regulatory feature in the formation of this core complex is the ability of syntaxin to flip between 2 different conformations (6). In the "closed" conformation, the N-terminus of syntaxin folds back onto itself, thus preventing SNARE assembly. In the "open" conformation, the $\mathrm{N}$-terminus of syntaxin moves away from the core $\alpha$ helix domain to allow interactions with other SNARE proteins. Several syntaxin-binding proteins have been identified as possible regulators of this conformational switch. Some of these are members of the Sec1p/Munc18 (SM) family of proteins (reviewed in ref. 7). The crystal structure of the neuronal Munc18-1/syntaxin1a complex demonstrates that the SM protein cradles syntaxin in its closed conformation, which supports the idea that SM proteins can differentiate among syntaxin conformations and/or facilitate the interconversion among conformations and possibly facilitate discrete syntaxin conformations, thus controlling vesicle fusion (8).
In a study reported in this issue of the JCI, Kanda and colleagues (9) generated mice deficient in the syntaxin4-binding protein Munc18c, which is involved in insulin-regulated GLUT4 trafficking (10). While the homozygous Munc18c deletion was lethal, Kanda and colleagues succeeded in isolating mesenchymal embryonic fibroblasts that could be induced to differentiate into insulin-responsive fat cells. Careful examination of insulin-stimulated GLUT4 trafficking within these cells revealed 2 novel findings. First, insulin-regulated GLUT4 trafficking can be divided into 2 discrete steps: (a) movement of GLUT4-containing vesicles close to the plasma membrane; and (b) docking and fusion of these vesicles with the plasma membrane. Second, Kanda et al. show that Munc18c is involved only in the docking and fusion step and this may be the major PI3K-Akt-regulated process in insulin-stimulated GLUT4 translocation.

\section{Step 1: trafficking to the cell surface}

At high insulin concentrations GLUT4 moves from a cytosolic location to the plasma membrane commensurate with increased glucose transport. In their study, Kanda et al. (9) show that in wild-type adipocytes, a submaximal insulin concentration stimulates accumulation of GSVs just beneath the cell surface that do not fuse, revealing 2 insulin-regulated steps in GLUT4 translocation that occur in series. The first, involving GSV trafficking to the plasma membrane, is highly insulin sensitive and is not inhibited by the PI3K inhibitor wortmannin, which is consistent with recent studies by others suggesting that this process is independent of the PI3K-Akt signaling pathway $(11,12)$.

Step 1 likely involves an interaction of GSVs with the cytoskeleton. Several recent studies have shown a role for microtubule-associated motor proteins $(13,14)$ as well as an actinassociated myosin in GLUT4 trafficking to the plasma membrane (11). The nature of the insulin-regulated association of GSVs with these structures remains to be defined. One candidate is phosphatidylinositol 3-phosphate (PI3P). Insulin has been shown to stimulate PI3P production in adipocytes in a wortmannin-insensitive manner and 


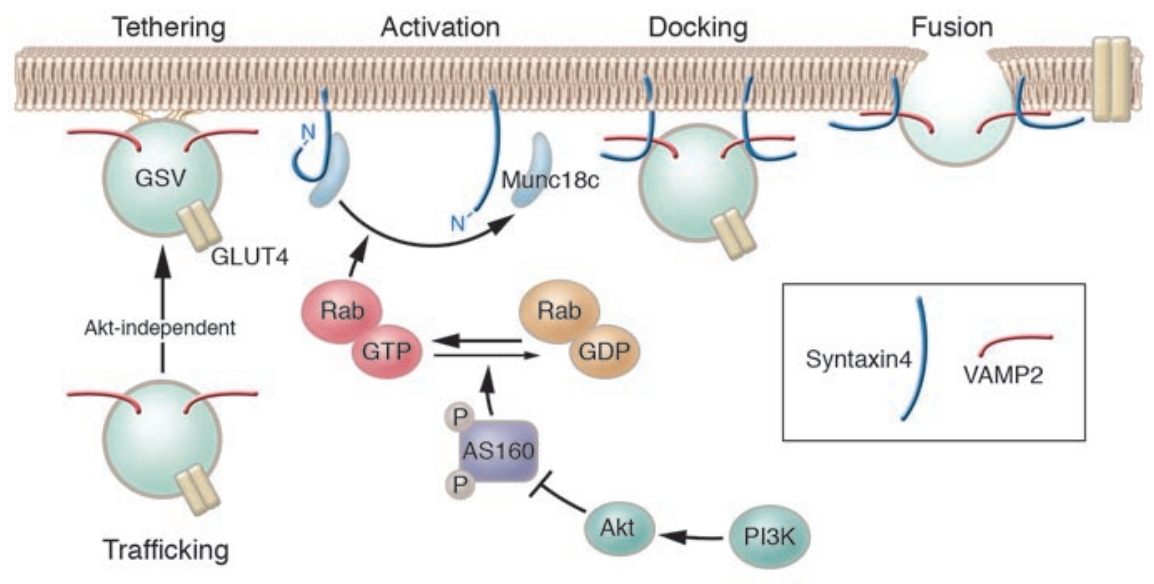

Figure 1

Insulin-stimulated GLUT4 translocation in adipocytes can be broken down into multiple steps. The first step involves trafficking of GLUT4-containing vesicles (GSVs) from an intracellular location close to the cell surface. As demonstrated by Kanda et al. in this issue of the $\mathrm{JCl}(9)$, this step is regulated by insulin in a PI3K-Akt-independent manner and occurs at low insulin concentrations. The second step involves multiple stages including: (a) tethering - a low-affinity interaction of GSVs with the plasma membrane; (b) activation - modification of the conformation of the Munc18c/syntaxin4 complex; (c) docking - a high-affinity interaction of GSVs with the plasma membrane; and (d) fusion - merging of the lipid bilayers of GSVs and the plasma membrane. The precise role of Munc18c in each of these stages is yet to be determined, but it is likely involved in both activation and docking. Importantly, the Munc18c-dependent stage also likely defines the major PI3K-Akt regulatory event in insulin-stimulated glucose transport under physiological conditions. The absence of Munc18c as described by Kanda et al. overcomes the PI3K-Akt regulation of GLUT4 trafficking, which suggests that Munc18c acts to somehow retain syntaxin4 in the "off" (closed) position and this clamp is removed in the presence of insulin, allowing syntaxin4 to move into the "on" position. This switch may be mediated by the activation of an as-yet-unidentified Rab protein, potentially involving Akt-dependent phosphorylation of the Rab GAP protein AS160 (18).

exogenous PI3P stimulates GLUT4 translocation in 3T3-L1 adipocytes (15). One cautionary note is that in both muscle and adipocytes freshly isolated from rodents under basal conditions, GSVs are often found very close to the plasma membrane $(16,17)$, which raises some doubt about the physiological significance of this step. One possible explanation for the observed proximity of the GSVs to the plasma membrane is that storage of GLUT4 in a deep intracellular location is either unique to cells in culture or related to the long preincubation of cells in the complete absence of insulin or other growth factors that is customary in experiments of this kind.

\section{Step 2: docking and fusion}

Extraordinarily, the complete lack of Munc18c had no discernible effect on insulin-stimulated glucose transport at high insulin concentrations (9). The surprising finding came when Kanda et al. examined Munc18c-null adipocytes stimulated with low insulin concentrations. In contrast to WT cells, in which they observed GLUT4 trafficking without fusion, Munc18c-null cells exhibited robust GLUT4 translocation as well as membrane insertion at $1-n M$ insulin concentrations, and neither of these effects were inhibited by wortmannin. This is a remarkable finding - placing the Munc18c/syntaxin4 dimer near the site of PI3K-Akt action! This finding is even more exciting in the context of recent studies by the Lienhard laboratory showing that one of the major Akt substrates in adipocytes is the $160-\mathrm{kDa}$ protein AS160 (also known as TBC4), which shows considerable homology with members of the Rab GTPase-activating protein (GAP) family $(18,19)$. Rab proteins are low-molecular-weight GTPases that play a fundamental role in vesicle docking and fusion (reviewed in ref. 20). Intriguingly, interactions have been described between each of the 4 SM proteins and Rab proteins in yeast genetic studies, which suggests that Rabs and SM proteins somehow work in tandem (reviewed in ref. 7). In view of this information, therefore, it is tempting to consider the proposed model shown in Figure 1. In the absence of insulin, Munc18c holds syntaxin 4 in an inactive closed conformation, thus rendering docking and fusion as a major rate-determining step in GLUT4's presentation at the cell surface. Insulin, through activation of Akt, results in phosphorylation of AS160, which somehow disables its GAP activity and results in GTP loading of its cognate Rab protein $(18,19)$. The GTP-loaded Rab may now bind to Munc18c, either directly or indirectly, weakening its hold on syntaxin4. Syntaxin 4 can then flip into a more active conformation, allowing GSVs to dock and fuse with the plasma membrane. Based on the work of Kanda et al. (9), one could predict that, in the absence of Munc18c, syntaxin 4 is constitutively open. Indeed the conformation undertaken by syntaxins when expressed in the absence of their cognate SM protein could represent one of the major differences among members of the syntaxin family. For example, deletion of the neuronal SM protein Munc18-1 results in a block in synaptic vesicle docking and fusion, possibly because the cognate target SNARE (syntaxin 1a) may constitutively undertake the closed conformation (see ref. 7).

The most important question regarding the model presented here (Figure 1) is, what is the identity of the Rab protein? So far, more than 60 different Rab isoforms have been identified in mammals, and the prediction is that each one is responsible for a discrete vesicle transport reaction. The recent identification of AS160, a Rab GAP protein, as an Akt substrate involved in GLUT4 trafficking should facilitate identification of the Rab protein because of the likelihood that these 2 proteins specifically interact. Once it is identified, we can begin to dissect how the Rab might interact with the Munc18c/syntaxin 4 complex. Second, are other regulated steps involved? Clearly, the encounter of GSVs with the plasma membrane may involve a number of steps aside from docking and fusion, and it will be necessary to design simple assays for each of these steps in order to dissect their molecular regulation. Third, is AS160 the only target of Akt in the GLUT4 translocation pathway? Finally, what controls the trafficking of GLUT4 to the plasma membrane (step 1)? It is likely that the docking and fusion step(s) play a more dominant role than the trafficking step in the regulated movement of GLUT4, as PI3K and Akt have been shown to be essential, and constitutively active versions of both are sufficient for GLUT4 translocation in adipocytes. With all these tools now available, one gets the sense that the holy grail of insulin action is within reach. 


\section{Acknowledgments}

Thanks to Robert Piper, Greg Cooney, and members of the James laboratory for invaluable discussions.

Address correspondence to: David E. James, Garvan Institute of Medical Research, Darlinghurst, Sydney, New South Wales 2010, Australia. Phone: 612-92958100; Fax: 61-2-92958201; E-mail: D.james@garvan.org.au.

1. Bryant, N.J., Govers, R., and James, D.E. 2002 Regulated transport of the glucose transporter GLUT4. Nat. Rev. Mol. Cell Biol. 3:267-277.

2. Whiteman, E.L., Cho, H., and Birnbaum, M.J. 2002. Role of Akt/protein kinase B in metabolism. Trends Endocrinol. Metab. 13:444-451.

3. Saltiel, A.R., and Pessin, J.E. 2002. Insulin signaling pathways in time and space. Trends Cell Biol. 12:65-71.

4. Chen, Y.A., and Scheller, R.H. 2001. SNARE-mediated membrane fusion. Nat. Rev. Mol. Cell Biol. 2:98-106.

5. Rizo, J., and Sudhof, T.C. 2002. Snares and
Munc18 in synaptic vesicle fusion. Nat. Rev. Neurosci. 3:641-653.

6. Dulubova, I., et al. 1999. A conformational switch in syntaxin during exocytosis: role of munc18. EMBO J. 18:4372-4382.

7. Toonen, R.F., and Verhage, M. 2003. Vesicle trafficking: pleasure and pain from SM genes. Trends Cell Biol. 13:177-186.

8. Misura, K.M., Scheller, R.H., and Weis, W.I. 2000. Three-dimensional structure of the neuronal-Sec1syntaxin 1a complex. Nature. 404:355-362.

9. Kanda, H., et al. 2005. Adipocytes from Munc18cnull mice show increased sensitivity to insulinstimulated GLUT4 externalization. J. Clin. Invest. 115:291-301. doi:10.1172/JCI200522681.

10. Tellam, J.T., et al. 1997. Characterization of Munc$18 \mathrm{c}$ and syntaxin- 4 in 3T3-L1 adipocytes. Putative role in insulin-dependent movement of GLUT-4. J. Biol. Chem. 272:6179-6186.

11. Bose, A., et al. 2004. Unconventional myosin Myo1c promotes membrane fusion in a regulated exocytic pathway. Mol. Cell. Biol. 24:5447-5458.

12. van Dam, E.M., Govers, R., and James, D.E. 2005 Akt activation is required at a late stage of insulininduced GLUT4 translocation to the plasma membrane. Mol. Endocrinol. In press.

13. Semiz, S., et al. 2003. Conventional kinesin KIF5B mediates insulin-stimulated GLUT4 movements on microtubules. EMBO J. 22:2387-2399.

14. Emoto, M., Langille, S.E., and Czech, M.P. 2001. A role for kinesin in insulin-stimulated GLUT4 glucose transporter translocation in 3T3-L1 adipocytes. J. Biol. Chem. 276:10677-10682.

15. Maffucci, T., Brancaccio, A., Piccolo, E., Stein, R.C., and Falasca, M. 2003. Insulin induces phosphatidylinositol-3-phosphate formation through TC10 activation. EMBO J. 22:4178-4189.

16. Slot, J.W., Geuze, H.J., Gigengack, S., James, D.E., and Lienhard, G.E. 1991. Translocation of the glucose transporter GLUT4 in cardiac myocytes of the rat. Proc. Natl. Acad. Sci. U. S. A. 88:7815-7819.

17. Slot, J.W., Geuze, H.J., Gigengack, S., Lienhard, G.E., and James, D.E. 1991. Immuno-localization of the insulin regulatable glucose transporter in brown adipose tissue of the rat. J. Cell Biol. 113:123-135.

18. Kane, S., et al. 2002. A method to identify serine kinase substrates. Akt phosphorylates a novel adipocyte protein with a Rab GTPase-activating protein (GAP) domain. J. Biol. Chem. 277:22115-22118.

19. Sano, H., et al. 2003. Insulin-stimulated phosphorylation of a Rab GTPase-activating protein regulates GLUT4 translocation. J. Biol. Chem. 278:14599-14602.

20. Zerial, M., and McBride, H. 2001. Rab proteins as membrane organizers. Nat. Rev. Mol. Cell Biol. 2:107-117.

\title{
Defining smooth muscle cells and smooth muscle injury
}

\author{
William M. Mahoney Jr. and Stephen M. Schwartz \\ Department of Pathology, University of Washington, Seattle, Washington, USA.
}

\begin{abstract}
For 3 decades, terms such as synthetic phenotype and contractile phenotype have been used to imply the existence of a specific mechanism for smooth muscle cell (SMC) responses to injury. In this issue of the JCI, Hendrix et al. offer a far more precise approach to examining the mechanisms of SMC responses to injury, focused not on general changes in phenotype but on effects of injury on a single promoter element, the CArG [CC(A/T) 6 GG] box, in a single gene encoding smooth muscle (SM) $\alpha$-actin (see the related article beginning on page 418). Since CArG box structures are present in some, but not all, SMC genes, these data suggest that we may be progressing toward establishing a systematic, molecular classification of both SMC subsets and the response of SMCs to different injuries.
\end{abstract}

Efforts to understand the response of arterial smooth muscle cells (SMCs) to injury have led to confusion, in part because of the as-yet-unconfirmed implication that terms such as dedifferentiation, synthetic phenotype, and phenotypic modulation refer to a specific, common mechanism. This issue of the JCI brings a major new perspective to

Nonstandard abbreviations used: $\mathrm{CArG}$, CC(A/T) ${ }_{6}$ GG; SM, smooth muscle; SMC, smooth muscle cell; SRF, serum response factor.

Conflict of interest: The authors have declared that no conflict of interest exists.

Citation for this article: J. Clin. Invest. 115:221-224 (2005). doi:10.1172/JCI200524272. this subject with a report by Hendrix et al. (1) that builds upon their recent findings (2).

Since the 1970s, most investigators assumed that the loss of properties (i.e., the loss of contractile capacity and the appearance of proteins associated with the extracelluar matrix) observed when SMCs adapted to culture used the same mechanisms required for the response of arterial SMCs to vascular injury, sometimes termed phenotypic modulation $(3,4)$. However, this theory of a common mechanism underlying the response of SMCs to multiple forms of injury in vivo is largely unsubstantiated. Since the molecular mechanism control- ling SMC differentiation and modulation in vivo was poorly understood, Hendrix et al. (1) examined the molecular control of smooth muscle (SM) $\alpha$-actin expression in response to injury. Their earlier studies showed that expression of SM $\alpha$-actin is regulated by promoter elements called CArG $\left[\mathrm{CC}(\mathrm{A} / \mathrm{T})_{6} \mathrm{GG}\right]$ boxes, which are bound by serum response factor (SRF) either alone or as a macromolecular complex including its specific cofactor, myocardin. Interestingly, cytoskeletal modulation regulates the SRFmyocardin interaction. Myocardin is bound by G-actin in the cytoplasm, and polymerization of actin releases myocardin, allowing it to travel to the nucleus. Once in the nucleus, it acts as a cofactor to enhance the binding of SRF to genes associated with cell replication and the dissociation from genes associated with SMC contractile proteins $(5,6)$. Therefore, there is a delicate balance between SMCs' need to respond to various stimuli and the availability of proteins to mediate these processes.

In the study by Hendrix et al. (1), transgenic mice with mutated CArG boxes were created as described in Figure 1. The effects 